CLINICAL STUDY

\title{
Impact of metformin and rosiglitazone treatment on glucose transporter 4 mRNA expression in women with polycystic ovary syndrome
}

\author{
Mojca Jensterle, Andrej Janez, Barbara Mlinar ${ }^{1}$, Janja Marc ${ }^{1}$, Janez Prezelj and Marija Pfeifer \\ Department of Endocrinology, Diabetes and Metabolic Diseases, University Medical Centre, Zaloška 7, SI-1000 Ljubljana, Slovenia and ${ }^{1}$ Department \\ of Clinical Biochemistry, Faculty of Pharmacy, University of Ljubljana, Ljubljana, Slovenia \\ (Correspondence should be addressed to M Pfeifer; Email: misa.pfeifer@kclj.si)
}

\begin{abstract}
Objective: The insulin-resistant state of the polycystic ovary syndrome (PCOS) was found to be associated with a decreased glucose transporter GLUT4 expression in the insulin target tissues. This study was performed to explore whether the well-known clinical, hormonal and metabolic efficacy of metformin or rosiglitazone treatment is reflected in the modulation of adipocyte GLUT4 mRNA expression in patients with PCOS.

Methods: We enrolled 35 women with PCOS. They received either metformin or rosiglitazone for 6 months. A history, blood samples for the measurement of androgens and s.c. adipose tissue samples were taken at baseline and end point. Quantification of GLUT4 mRNA expression in adipose tissue was performed using real-time quantitative PCR. Homeostasis model assessment $\left(\mathrm{HOMA}_{\mathrm{IR}}\right)$ score calculation was applied as a measure for insulin resistance (IR).

Results: GLUT4 mRNA expression in adipose tissue increased significantly in both groups $(P<0.001)$. The increase was more pronounced in the rosiglitazone group $(P=0.040)$. There was a statistically significant improvement of $\mathrm{HOMA}_{\mathrm{IR}}$ in both groups $(P=0.008)$. After treatment, frequencies of menstrual bleeding were significantly higher $(P<0.001)$ and serum total testosterone levels significantly lower in both groups $(P=0.001)$.

Conclusions: A 6-month therapy with insulin sensitizers resulted in marked improvement in adipose tissue GLUT4 mRNA expression in PCOS patients, rosiglitazone being more effective when compared with metformin. The augmentation of the insulin signal transduction was accompanied by a significant improvement of $\mathrm{HOMA}_{\mathrm{IR}}$, menstrual pattern and androgen profile.
\end{abstract}

European Journal of Endocrinology 158 793-801

\section{Introduction}

Polycystic ovary syndrome (PCOS) is a common endocrinopathy of complex and multifactorial aetiology characterized by hyperandrogenism and chronic anovulation (1). It affects $4-10 \%$ women of reproductive age and is one of the most common causes of anovulatory infertility (2). Over the last 20 years, it has been widely recognized that in addition to endocrine and reproductive abnormalities, most women with PCOS demonstrate metabolic disturbances. Insulin resistance (IR) is the most significant and highly prevalent among these. Overall, 50-60\% of PCOS patients suffer from IR $(3,4)$ compared with a prevalence of IR in the general population of $10-25 \%$, depending on methods applied and the population's mean body weight (5-7).

The IR, which is intrinsic to the syndrome and independent of obesity, is poorly understood. Several laboratories have investigated the cellular mechanisms underlying IR in PCOS (8-13). The adipose tissue seems to play an important role in the development and maintenance of the condition. The cellular mechanisms underlying IR in PCOS are secondary to an early postreceptor defect in insulin-signalling cascade with a decreased downstream signal transduction in the face of normal insulin binding (12). The distal aspects of insulin signalling, beyond insulin receptor action and phosphorylation, have been less well explored. It was reported that the amount of adipocyte GLUT4 transporters was lower in PCOS patients than in controls and was independent of body mass index (BMI) (13).

The most significant advance in the understanding of PCOS has been the recognition that IR accompanied by compensatory hyperinsulinemia is a key component in the pathogenesis of the syndrome (14). This finding has led to the application of insulin-sensitizing drugs, metformin and thiazolidinediones (TZDs), as a treatment option for this disorder. Metformin is the most extensively studied insulin-sensitizing agent for the treatment of women with PCOS. It inhibits hepatic glucose production 
and enhances peripheral tissue sensitivity to insulin, necessitating a lower insulin concentration and thereby probably reducing the androgen production of theca cells in the ovaries (15). Metformin also appears to suppress ovarian steroidogenesis directly $(16,17)$. Some investigators have reported increased sex hormone binding globulin (SHBG) levels with metformin, decreasing free androgen bioavailability (18). Many studies have shown that metformin reduces plasma androgen levels and improves parameters of hyperinsulinism $(19,20)$. Several randomized trials and meta-analyses have demonstrated its efficacy in increasing ovulation rates when used as monotherapy and increasing ovulation and pregnancy rates when used in conjunction with clomiphene citrate (19-21). The recent Cochrane review compared metformin with the oral contraceptives (OCP) in PCOS. Limited data demonstrated no evidence of difference in effect between the metformin and the OCP on hirsutism and acne. Metformin was less effective than the OCP in improving menstrual pattern and in reducing serum androgen levels and more effective than the OCP in reducing fasting insulin and not increasing triglyceride levels (22). However, the effects of metformin on hirsutism (23), weight reduction (19-21, 23, 24) and lipids $(25,26)$ in PCOS subjects remain controversial. The actual protection from cardiovascular morbidity and mortality has yet to be demonstrated.

Currently, there are limited data on the use of TZDs in PCOS. TZDs exert their insulin-sensitizing actions through the peroxisome proliferator-activated receptor- $\gamma$ found in a number of tissues including the liver, the skeletal muscle and the adipose tissue. They increase insulin sensitivity without increasing insulin secretion through activation of multiple genes, including the up-regulation of glucose transporters (GLUTs). TZDs increase the uptake and utility of glucose in peripheral tissues and decrease hepatic gluconeogenesis, promote adipocyte differentiation, in the adipose tissue, leading to the production of smaller, more insulin-sensitive fat cells (27). They redistribute fat from the visceral fat compartment, which is strongly associated with IR and cardiovascular risk, to the s.c. fat tissue, which is not associated with a cardiovascular risk $(28,29)$. They also affect ovarian steroid synthesis directly by inhibiting enzymes $3 \beta$-hydroxysteroid dehydrogenase and P450c17- $\alpha(30)$. Findings report increased SHBG with TZDs (31). Troglitazone that is now unavailable, due to hepatic toxicity, is the most researched agent in PCOS. It produced significant endocrine, metabolic and ovulatory benefits (32-34). Rosiglitazone, the representative of a new generation of TZDs, has been shown to increase the ovulation rates, reduce the hyperandrogenism and improve the metabolic profile (35-40).

Despite the presence of several well-documented favourable reproductive and metabolic effects of insulin sensitizers in PCOS population, their tissue-specific cellular effects in PCOS are, however, less clear. The purpose of our study was to explore whether the clinical, hormonal and metabolic efficacy of metformin and rosiglitazone treatment is reflected in a modulation of the gene expression for the most important glucose transporter GLUT4 in subcutaneous adipocytes from women with PCOS.

\section{Subjects and methods}

\section{Study population}

We recruited 35 women with PCOS as classified according to the National Institute of Child Health and Human Development (NICHD) criteria (41). We have chosen the NICHD criteria because they better define the population of PCOS patients that are at higher risk for IR and cardiometabolic complications than the Rotterdam criteria do (42). All subjects gave their written informed consent before entering the study, which was conducted in accordance with the Declaration of Helsinki and approved by the national ethical committee. The baseline characteristics of the patients are presented in Table 1. Clinical hyperandrogenism was defined by the presence of hirsutism, represented by a modified Ferriman - Gallwey score of 7 or more, the persistence of acne during the third decade of life or later, or the presence of androgenic alopecia. No attempts were made to grade the severity of acne or alopecia. Hyperandrogenemia was defined as a total or free testosterone, androstenedione, and/or DHEA sulphate (DHEAS) level above the 95th percentile of normal population values. Menstrual dysfunction was defined by more than six cycles with a length of more than 35 days (oligomenorrhea), and/or when the patient had not had any menstrual bleeding for 3 consecutive months (amenorrhea) during the previous year. We took a history of menstrual bleeding for the period of 6 months before starting the treatment and followed each menstrual bleeding during the treatment. All patients had normal serum prolactin concentrations and thyroid function tests. Possible Cushing's syndrome or congenital (nonclassic) adrenal hyperplasia was excluded (41). Additional exclusion criteria were type 1 or type 2 diabetes mellitus, a significant cardiovascular or hepatic disease, and the use of medications known or suspected to affect reproductive or metabolic functions, within 60 days prior to study entry. None of the patients had ever taken insulinsensitizing drugs prior to the study. The control group (Controls) included 12 age-matched healthy women without clinical or laboratory evidence of PCOS and with normal insulin sensitivity (age (years; mean \pm s.D.) $23.5 \pm 0.7$, BMI $\left(\mathrm{kg} / \mathrm{m}^{2}\right) 20.9 \pm 0.73$, homeostasis model assessment $\left.\left(\mathrm{HOMA}_{\mathrm{IR}}\right) 0.85 \pm 0.15\right)$. They were not on any medication that could affect hormonal status.

\section{Experimental protocol}

The patients were randomly allocated to a 6-month treatment with either metformin (MET) $850 \mathrm{mg}$ twice daily or rosiglitazone (ROSI) $4 \mathrm{mg}$ once a day for 


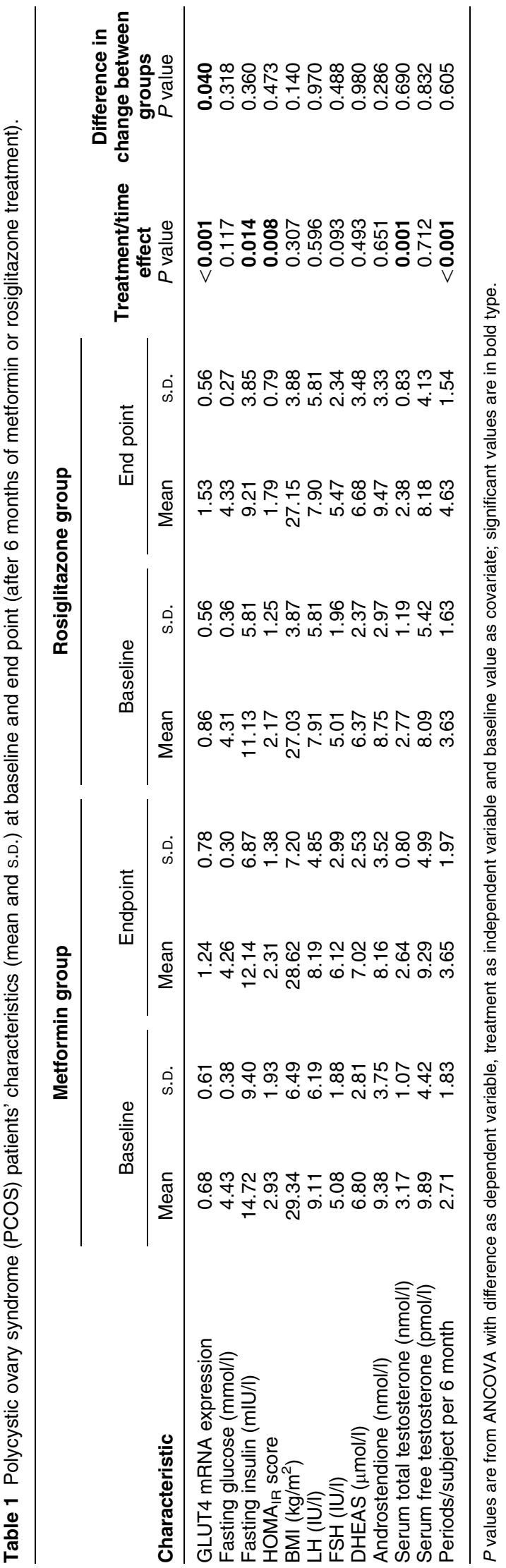

6 months. As a method of randomization, the RAND programme in Excel was used. The MET group included 18 women (age (years) $22.9 \pm 4.5$ ). The ROSI group included 17 women (age (years) 25.2 \pm 4.8 ). At baseline and end point of the study, they underwent a physical examination. A fasting blood sample was drawn for determination of glucose, insulin and other observation parameters followed by a standard $75 \mathrm{~g}$ oral glucose tolerance test (OGTT). All the blood samples were centrifuged and the separated serum was kept frozen at $-40{ }^{\circ} \mathrm{C}$ until the time of the assay. Subcutaneous adipose tissue samples were obtained from the sub-umbilical abdominal region under local anaesthetic with $2 \%$ lidocaine. A sterile needle $(3 \mathrm{~cm} \times$ $2 \mathrm{~mm}$ ) attached to a $20 \mathrm{ml}$ sterile plastic syringe was inserted through the skin and the adipose tissue was drawn into the syringe by suction (43). The adipose tissue was immediately frozen in liquid nitrogen and stored at $-70{ }^{\circ} \mathrm{C}$ until analyzed. Since the majority of patients suffered oligomenorrhea or amenorrhea during the previous year, the assessment of the subjects was not based on any specific stage of the menstrual cycle. The women were advised to use barrier contraception, instructed not to modify their usual eating habits throughout the study, and asked to report any side effects during the treatment. In addition, safety parameters (haematology, liver and renal function and serum electrolytes) were assessed before and at 2-month intervals during the study.

\section{Assays}

Glucose levels were determined using a standard laboratory reference method (glucose oxidase method; Roche Hitachi 917). Luteinizing hormone (LH) and follicle-stimulating hormone (FSH) were determined using an immunometric assay (Diagnostic Products Corporation, Los Angeles, CA, USA). Androstenedione and DHEAS were measured by specific double antibody RIA using 125 I-labelled hormones (Diagnostic Systems Laboratories, Webster, TX, USA). Total and free testosterone levels were measured by coated tube RIA (DiaSorin, S. p. A, Salluggia, Italy and Diagnostic Products Corporation, respectively). Insulin was determined by IRMA (Biosource Europe S.A., Nivelles, Belgium). Intraassay variations ranged from 1.6 to $6.3 \%$ and inter-assay variations ranged from 5.8 to $9.6 \%$ for the applied methods. Pre- and post-treatment samples from each patient were assayed in the same assay run.

\section{Determination of IR}

$\mathrm{HOMA}_{\mathrm{IR}}$ score calculation was applied as a measure for IR. The estimate of IR by HOMA IR score was calculated with the following formula: fasting serum insulin $(\mathrm{mU} / \mathrm{l}) \times$ fasting plasma glucose $(\mathrm{mmol} / \mathrm{l}) / 22.5$ (44). HOMA $_{\text {IR }}$ score values 2.0 were considered as a cut-off point for IR as published previously (45). 
Quantification of GLUT4 mRNA expression by real-time quantitative PCR in adipose tissue

Total RNA from adipose tissue was extracted using RNeasy Lipid Tissue Mini Kit (Qiagen). The integrity and concentration of the RNA were verified by the analysis on the Agilent 2100 Bioanalyzer. RT into cDNA was performed using TaqMan RT Reagents (Applied Biosystems, Foster City, CA, USA). Real-time PCR was performed on ABI PRISM 7000 Sequence Detection System (Applied Biosystems) using TaqMan technology. Each reaction mixture $(25 \mu \mathrm{l})$ contained $5 \mathrm{ng}$ RNA converted to cDNA, $1 \times$ TaqMan Universal PCR Master Mix and $1 \times$ primers and probes for GLUT4 (inventoried assay) and phosphoglycerate kinase (PGK; TaqMan Endogenous Controls, both purchased from Applied Biosystems). PGK was chosen as endogenous control among 11 housekeeping genes upon analysis on TaqMan Endogenous Control Plate (Applied Biosystems). GLUT4 probe was labelled with 6-carboxyfluorescein (FAM), and PGK probe with VIC fluorescent dye. Each experiment was run with the samples tested in triplicate and with the non-template control. The TaqMan PCR conditions were as follows: $2 \mathrm{~min}$ at $50^{\circ} \mathrm{C}$, $10 \mathrm{~min}$ at $95^{\circ} \mathrm{C}$ and then 40 cycles each of $15 \mathrm{~s}$ at $95^{\circ} \mathrm{C}$ and $1 \mathrm{~min}$ at $60^{\circ} \mathrm{C}$ on MicroAmp Optical 96-Well Reaction Plate (Applied Biosystems). Standard curve for GLUT4 cDNA and PGK cDNA was generated by serial dilution of randomly chosen cDNA samples for each plate. Thus, mRNA concentrations were calculated from the cycle number where the fluorescence crosses the threshold $\left(C_{\mathrm{t}}\right)$. The expression of GLUT4 gene was then normalized by dividing of the GLUT4 mRNA concentration by PGK mRNA concentration for each sample.

\section{Statistical analysis}

Normality was assessed graphically using histograms and Q-Q plots. Because no obvious deviations from normality were observed, the parametric statistical methods were used. Pre-treatment and post-treatment data were expressed as mean and S.D. Differences between the treatment groups at baseline were tested using Student's independent two-tailed $t$-test. Pre-treatment differences in GLUT4 mRNA expression between the women with PCOS (separately for the patients before and after treatment) and controls were also tested using Student's independent two-tailed $t$-test. Because of curvilinearity, bivariate associations were assessed using Spearman's rank correlation $(\rho)$. Analysis of covariance (ANCOVA) approach was used to compare the treatment change between the ROSI and the MET groups (via group effect) while adjusting the outcomes for possible baseline imbalances, and to test for the effect of being treated (via intercept from the model with centred predictors). A value of $P<0.05$ was considered statistically significant.
Statistical analyses were performed with SPSS for Windows 13.0 software (SPSS Inc., Chicago, IL, USA).

\section{Results}

After the initial inclusion of 35 patients, 33 (94.3\%) finished the trial according to the protocol. Two women, one from each group, were excluded due to protocol violation.

\section{Baseline results}

The mean \pm s.D. values for the observed parameters at baseline are reported in Table 1. As expected because of randomization, there were no significant differences at baseline in any of the parameters between the treatment groups ( $P$ values not reported).

\section{Changes in anthropometric and circulating variables}

Only minor and statistically non-significant changes were observed in the BMI values in both groups after the treatment intervention (Table 1). OGTT did not reveal an impaired glucose tolerance (IGT) in any of the patients studied in the ROSI group (neither at baseline nor at end point). In the MET group, two patients were found to have IGT at baseline. After treatment, the IGT was still observed in the same two patients. At baseline, $47 \%$ of patients in the MET group and $50 \%$ of patients in the ROSI group were insulin sensitive according to their $\operatorname{HOMA}_{\mathrm{IR}}$ score $(<2)$. After 6 months of treatment, $\mathrm{HOMA}_{\mathrm{IR}}<2$ was found again in $47 \%$ of patients in the MET group and in $56 \%$ of patients in the ROSI group. Overall, HOMA ${ }_{I R}$ score values significantly decreased in both groups $(P=0.008$ for the treatment/time effect). The improvement in both groups was predominantly driven by a decrease in fasting insulin values ( $P=0.014$ for the treatment/time effect). Furthermore, administration of both drugs was associated with a significant decrease in serum total testosterone $(P=0.001$ for effect of time), on average by $16.7 \%$ (MET) and $14.1 \%$ (ROSI) respectively (Fig. 1). No change over time occurred in the remaining circulating variables (fasting glucose, $\mathrm{LH}$, FSH, androstenedione, DHEAS and free testosterone).

\section{Changes in menstrual pattern}

Both treatment interventions resulted in an improvement of patients' menstrual pattern $(P<0.001$ for the treatment/time effect). Menstrual frequency increased from 0.45 to 0.61 cycles per month (2.71-3.65 per 6 months) on average in the MET group and from 0.62 to 0.77 per month (3.63-4.63 per 6 months) in the ROSI group (Fig. 2). One woman in the MET group with no menstruation during the previous 6 months before treatment had two menstruations during the treatment time. The same improvement was achieved in two women 

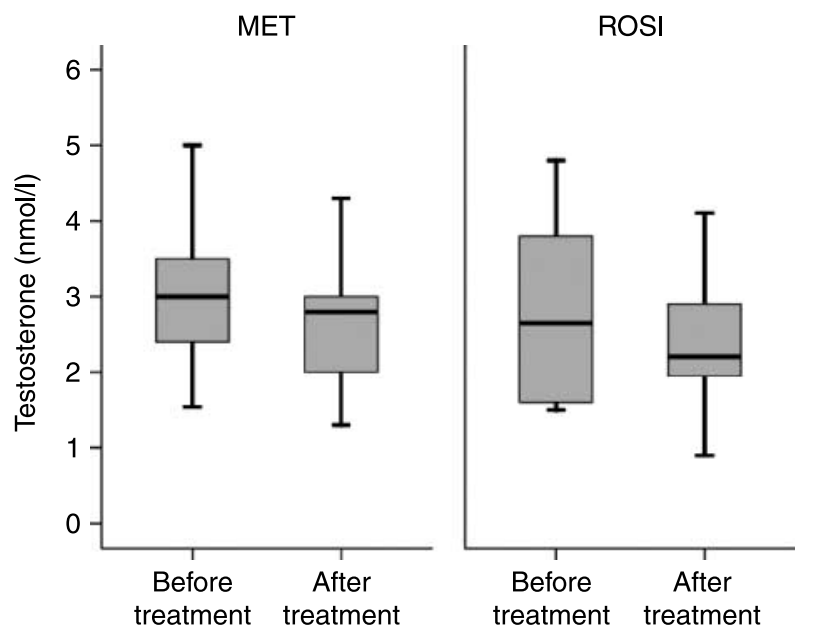

Figure 1 Distribution of the serum total testosterone at baseline and end point by the treatment groups $(P=0.001$ for the treatment/time effect), MET, the group on metformin and ROSI, the group on rosiglitazone.

in the ROSI group. Three (19\%) women in the MET and six (38\%) women in the ROSI group with previously irregular cycles (of an average length of 35 days) achieved regular menses (an average cycle length of 28 days). Overall, 12 (71\%) women in the MET group and $13(81 \%)$ in the ROSI group experienced an improvement in their menstrual pattern after 6 months of treatment.

\section{Biopsy results}

As expected, there was a negative association between BMI and adipose tissue GLUT4 expression in the patients. It was less pronounced at baseline (Spearman

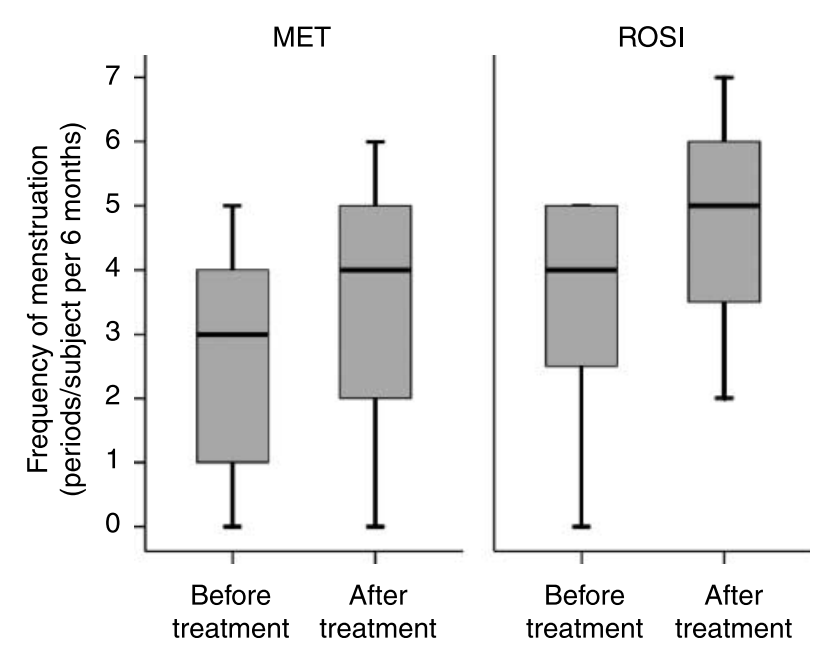

Figure 2 Distribution of menstruation frequency at baseline and after 6 months of treatment by the treatment groups $(P<0.001$ for the treatment/time effect), MET, the group on metformin and ROSI, the group on rosiglitazone. $\rho=-0.18, P=0.355)$ than after treatment, when it became statistically significant (Spearman $\rho=-0.42$, $P=0.022$ ). GLUT4 mRNA expression in adipocytes increased significantly with treatment in both groups $(P<0.001$ for the treatment/time effect $)$. The increase was more pronounced in the ROSI group $(P=0.04$ from ANCOVA; Fig. 3). Baseline $\mathrm{HOMA}_{\mathrm{IR}}$ was positively associated with the change in GLUT4 expression (Spearman $\rho=0.50, P=0.005$ ). However, after treatment, the values of GLUT4 mRNA were still significantly lower in both groups when compared with the control group (Fig. 4).

Both drugs were well tolerated and a few clinically minor adverse events did not lead to discontinuation of the treatment in any of the patients. Three subjects in the ROSI group complained about mild headaches. In the MET group, four subjects had temporary mild gastrointestinal problems. No elevation of liver enzymes was found and no case of oedema was reported in the ROSI group.

\section{Discussion}

Our study showed that clinical, hormonal and metabolic improvements with metformin and rosiglitazone treatment are reflected in an increased gene expression of the most important glucose transporter GLUT4 in the subcutaneous adipose tissue of women with PCOS. To our knowledge, this is the first trial to compare the efficacy of metformin and rosiglitazone on adipocyte GLUT4 mRNA expression in PCOS population.

Before the treatment, we found a significantly lower adipocyte GLUT4 mRNA expression in PCOS women when compared with age-matched healthy individuals, which is in accordance with the Rosenbaum's study (13). After the treatment, GLUT4 mRNA expression increased significantly in adipocytes of both treatment groups, the increase being significantly more pronounced in the ROSI group. There was a positive correlation between the baseline $\mathrm{HOMA}_{\mathrm{IR}}$ score and the increase in GLUT4 expression with treatment, indicating that insulin sensitizers seem to be more potent in subjects with more pronounced IR. However, although GLUT4 mRNA expression was nearly doubled after the treatment in both groups, the post-treatment values did not reach the values of the healthy controls. Nevertheless, it needs to be pointed out that the normal weight controls were not BMI matched with overweight PCOS intervention groups. Therefore, it is practically impossible to differentiate the effects of obesity or PCOS status per se on the lower post-treatment values of GLUT4 mRNA expression in both PCOS intervention groups in comparison with higher GLUT4 mRNA expression in lean controls. A study design problem in this context originated in insufficient number of the BMI-matched healthy volunteers willing to be exposed to invasive biopsy procedure. 

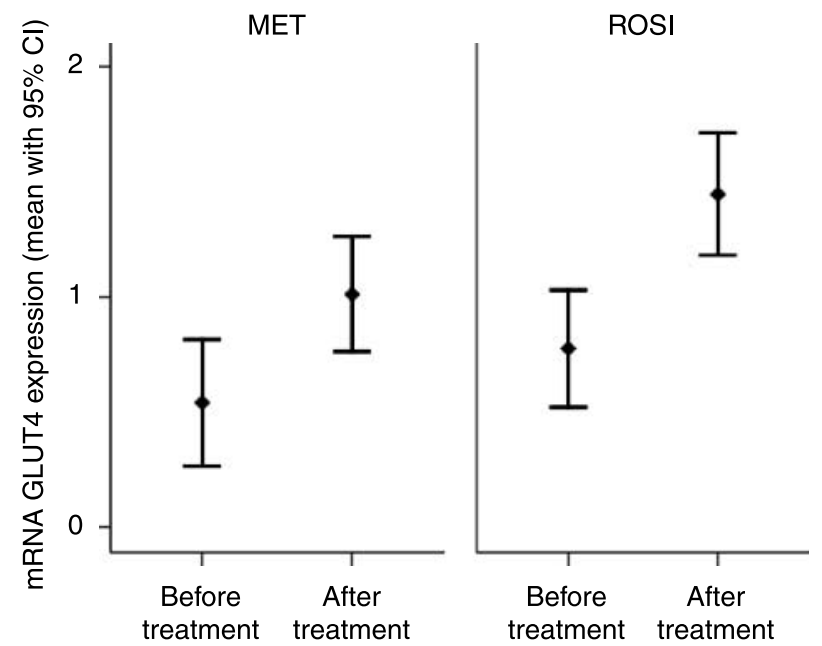

Figure 3 GLUT4 mRNA expression in the adipose tissue of PCOS patients at baseline and after 6 months of treatment by the treatment groups $(P=0.040$ for the difference in treatment effect between groups), MET, the group on metformin and ROSI, the group on rosiglitazone.

Since GLUT4 expression is the final step in the insulin-signalling cascade leading to glucose internalization into the cell, its improvement appears to imply an overall improvement of insulin sensitivity. In agreement with this, we noticed a statistically significant improvement of IR as measured by HOMA IR score in MET and ROSI groups. Additionally, we observed improvements of clinical and hormonal parameters in both treatment arms. The interval between menstrual periods shortened significantly implying better ovarian function. Serum total testosterone levels significantly decreased, demonstrating that both rosiglitazone and metformin

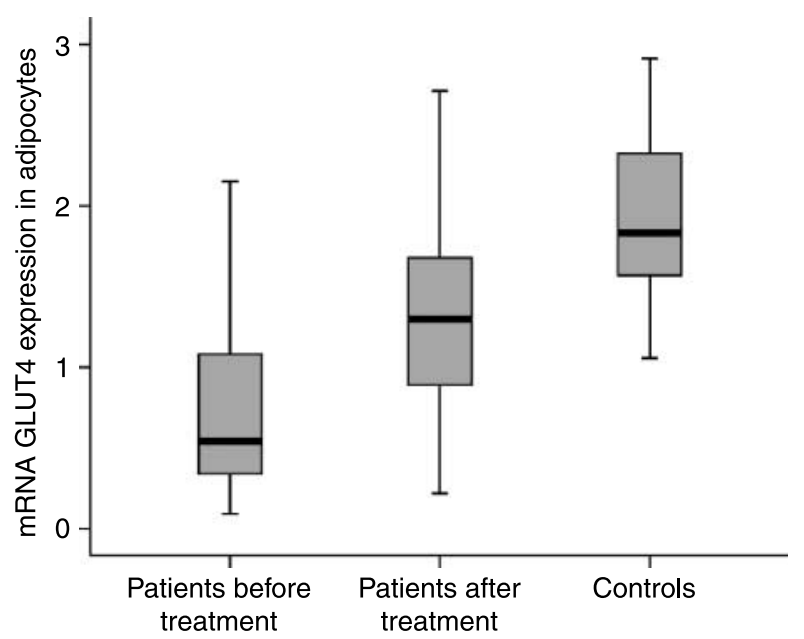

Figure 4 Distribution of GLUT4 mRNA expression in the adipose tissue of women with PCOS at baseline, after 6 months of treatment with metformin or rosiglitazone, and in the control group (independent sample $t$-test: $P<0.001$ for patients before treatment versus controls; $P=0.015$ for patients after treatment versus controls). effectively reduced androgen secretion. No drug was found to be superior to the other, as far as metabolic, menstrual and hormonal aspects were concerned.

At present, there are limited data in the literature on the use of metformin versus rosiglitazone on these parameters in PCOS. Baillargeon et al. suggested that metformin and rosiglitazone increase ovulatory frequency and ameliorate hyperandrogenemia even in lean women with PCOS who appear to have normal insulin sensitivity (46). Similarly, a clinically meaningful improvement in menstrual pattern and a significant reduction of testosterone concentration were observed in our study population who was not severely insulin resistant. Another study by Mitkov et al. reported a greater reduction in androgens in metformin treatment arm, while rosiglitazone was more potent in improving IR and hyperinsulinemia (47). Legro et al. showed that rosiglitazone was more efficient than metformin in reducing elevated insulin and androgen levels in an obese PCOS population (48). In our patients, both drugs were equally effective in reducing androgen levels, fasting insulin levels and $\mathrm{HOMA}_{\mathrm{IR}}$ score, while an improvement of GLUT4 expression was more pronounced with rosiglitazone treatment. However, considering a paucity of data from randomized controlled studies (RCTs), it is not clear whether rosiglitazone is more potent than metformin in improving the clinical and biochemical features of PCOS. Further research involving RCTs with larger numbers of patients is needed before any recommendation can be made on the usefulness of these agents in the treatment of PCOS (49) and to determine the mechanisms implied in their treatment effects.

The augmentation of insulin sensitivity was probably the most important mechanism by which metformin and rosiglitazone improved menstrual and hormonal profile in our PCOS patients, even though they were not considered severely insulin resistant. Potentially, metformin and rosiglitazone can even be efficient in PCOS women with normal insulin sensitivity, implying an additional mechanism for their efficacy in improving menstrual cyclicity and androgen profile in our patients. Several lines of evidence support the notion that TZDs have a direct effect on insulin signalling and on ovarian steroidogenesis apart from an improvement of insulin sensitivity (30, 5054). By its direct effects on the ovary, rosiglitazone may inhibit the steroidogenic enzymes and contribute to the improvement of menstrual pattern and androgen profile. The information on metformin actions on tissues other than hepatic tissues varies $(16,17,54,55)$, and the question of direct effects of metformin on ovarian steroidogenesis remains to be resolved.

Regarding translation of our data to clinical use in PCOS women, it needs to be pointed out, however, that the issue of teratogenicity with TZDs, based on animal studies, is a real concern for the clinical use of rosiglitazone in women of reproductive age. Because of an uncertain safety profile in pregnancy, rosiglitazone 
should not be prescribed to women wishing to conceive. Alternatively, many studies have confirmed apparent safety of metformin, with no teratogenicity and even with a potential for reducing the risk of miscarriage and gestational diabetes in PCOS women (56). Therefore, the administration of metformin is applicable in younger women of reproductive age, whereas the potential use of rosiglitazone may be of benefit to the older PCOS women who are at higher risk of cardiometabolic complications.

Before conclusion, the limitations of our study should be taken into consideration. This is an intervention study with relatively small sample size. Even though the number of PCOS patients was limited by organizational and funding constraints, and mostly by the invasiveness of adipose tissue collection, it was still sufficient for medium standardized effect size. Furthermore, IR was not assessed by the gold standard method, the euglycemic hyperinsulinemic clamp technique, or by area under the curve for glucose and insulin during OGTT in order to better characterize IR. However, there is no consensus for the assessment of IR in PCOS and there are strengths and weaknesses in the most known methods. In addition, a specific BMI was not an inclusion criterion; therefore, we studied patients with a broad spectrum of BMI. It is possible that the potential differences in IR for lean and overweight PCOS subjects would introduce additional variability into the results, but the subgroup analysis of lean and overweight women is not warranted given the small sample size.

In conclusion, the 6-month treatment with rosiglitazone and metformin of PCOS women, with a broad spectrum of BMI who were not considered severely insulin resistant, resulted in marked improvements in GLUT4 mRNA expression in adipose tissue. The augmentation of the insulin signal transduction was accompanied by statistically significant improvement in $\mathrm{HOMA}_{\mathrm{IR}}$ score, menstrual frequency and a significant reduction in serum total testosterone concentration. Whereas rosiglitazone produced a greater increase in adipocyte GLUT4 mRNA expression when compared with metformin, suppression of $\mathrm{HOMA}_{\mathrm{IR}}$, serum testosterone levels and the improvement of menstrual pattern were comparable in both groups. Further RCTs are required to compare the efficacy of metformin versus rosiglitazone in PCOS and to determine whether direct tissue-specific mechanisms or indirect metabolic mediators are implied in their treatment effects.

\section{Acknowledgements}

We are grateful to Alenka Roš, RN, for assistance with the assay procedures and for performing anthropometric measurements. We appreciate the support of Prof. Andreas Pfutzner from IKFE Institute for Clinical Research and Development, Mainz, Germany, with whom we had invaluable discussions, and we thank
Gaj Vidmar, PhD, from the Institute of Biomedical Informatics in Ljubljana for help with data analysis and technical writing. The study was supported by Slovenian Research Agency, Research Program No. P3-0298.

\section{References}

1 Franks S. Polycystic ovary syndrome. New England Journal of Medicine $1995333853-856$.

2 Azziz R, Woods KS, Reyna R, Key TJ, Knochenhauer ES \& Yildiz BO. The prevalence and features of the polycystic ovary syndrome in an unselected population. Journal of Clinical Endocrinology and Metabolism 200489 2745-2749.

3 Dunaif A, Segal KR, Futterweit W \& Dobrjansky A. Profound peripheral insulin resistance, independent of obesity, in polycystic ovary syndrome. Diabetes 1989381165.

4 Legro RS, Finegood D \& Dunaif A. A fasting glucose to insulin ratio is a useful measure of insulin sensitivity in women with polycystic ovary syndrome. Journal of Clinical Endocrinology and Metabolism $1998832694-2698$.

5 Burchfiel CM, Curb JD, Arakaki R, Abbott RD, Sharp DS, Rodriguez BL \& Yano K. Cardiovascular risk factors and hyperinsulinemia in elderly men: the Honolulu Heart Program. Annals of Epidemiology $1996 \mathbf{6} 490-497$.

6 Ferrannini E, Natali A, Bell P, Cavallo-Perin P, Lalic N \& Mingrone G. Insulin resistance and hypersecretion in obesity. European Group for the Study of Insulin Resistance EGIR. Journal of Clinical Investigations $1997 \mathbf{1 0 0} 1166-1173$.

7 Bonora E, Kiechl S, Willeit J, Oberhollenzer F, Egger G, Targher G, Alberiche M, Bonadonna RC \& Muggeo M. Prevalence of insulin resistance in metabolic disorders: the Bruneck Study. Diabetes 199847 1643-1649.

8 Dunaif A, Segal KR, Shelley DR, Green G, Dobrjansky A \& Licholai T. Evidence for distinctive and intrinsic defects in insulin action in polycystic ovary syndrome. Diabetes $1992 \mathbf{4 1}$ 1257-1266.

9 Ciaraldi TP, el Roeiy A, Madar Z, Reichart D, Olefsky JM \& Yen SSC. Cellular mechanisms of insulin resistance in polycystic ovarian syndrome. Journal of Clinical Endocrinology and Metabolism 1992 75 577-583.

10 Dunaif A, Xia J, Book CB, Schenker E \& Tang Z. Excessive insulin receptor serine phosphorylation in cultured fibroblasts and in skeletal muscle. A potential mechanism for insulin resistance in the polycystic ovary syndrome. Journal of Clinical Investigations $199596801-810$.

11 Li M, Youngren JF, Dunaif A, Goldfine ID, Maddux BA, Zhang BB \& Evans JL. Decreased insulin receptor (IR) autophosphorylation in fibroblasts from patients with PCOS: effects of serine kinase inhibitors and IR activators. Journal of Clinical Endocrinology and Metabolism $2002 \mathbf{8 7} 4088-4093$.

12 Venkatesan AM, Dunaif A \& Corbould A. Insulin resistance in polycystic ovary syndrome: progress and paradoxes. Recent Progress in Hormone Research 200156 295-308.

13 Rosenbaum D, Haber RS \& Dunaif A. Insulin resistance in polycystic ovary syndrome: decreased expression of GLUT-4 glucose transporters in adipocytes. American Journal of Physiology 1993264 197-202.

14 Dunaif A. Insulin resistance and the polycystic ovary syndrome: mechanism and implications for pathogenesis. Endocrine Reviews 199718 774-800.

15 Ehrmann DA. Polycystic ovary syndrome. New England Journal of Medicine 2005352 1223-1236.

16 Attia GR, Rainey WE \& Carr BR. Metformin directly inhibits androgen production in human thecal cells. Fertility and Sterility $200176517-524$. 
17 Mansfield R, Galea R, Brincat M, Hole D \& Mason H. Metformin has direct effects on human ovarian steroidogenesis. Fertility and Sterility 2003 79 956-962.

18 Nawrocka J \& Starczewski A. Effects of metformin treatment in women with polycystic ovary syndrome depends on insulin resistance. Gynecological Endocrinology 200723 231-237.

19 Harborne L, Fleming R, Lyall H, Norman J \& Sattar N. Descriptive review of the evidence for the use of metformin in polycystic ovary syndrome. Lancet $2003 \mathbf{3 6 1} 1894-1901$.

20 Lord JM, Flinght IH \& Norman RJ. Metformin in polycystic ovary syndrome: systematic review and meta-analysis. BMJ 2003327 951-953.

21 Legro RS, Barnhart HX, Schlaff WD, Carr BR, Diamond MP, Carson SA, Steinkampf MP, Coutifaris C, McGovern PG, Cataldo NA, Gosman GG, Nestler JE, Giudice LC, Leppert PC \& Myers ER. Cooperative Multicenter Reproductive Medicine Network. Clomiphene, metformin, or both for infertility in the polycystic ovary syndrome. New England Journal of Medicine $2007356551-566$.

22 Costello MF, Shrestha B, Eden J, Johnson NP \& Sjoblom P. Metformin versus oral contraceptive pill in polycystic ovary syndrome: a Cochrane review. Human Reproduction 200722 1200-1209.

23 Harborne L, Fleming R, Lyall H, Sattar N \& Norman J. Metformin or antiandrogen in the treatment of hirsutism in polycystic ovary syndrome. Journal of Clinical Endocrinology and Metabolism 2003 $884116-4123$.

24 Harborne LR, Sattar N, Norman JE \& Fleming R. Metformin and weight loss in obese women with polycystic ovary syndrome: comparison of doses. Journal of Clinical Endocrinology and Metabolism 200590 4593-4598.

25 Tang T, Glanville J, Hayden CJ, White D, Barth JH \& Balen AH. Combined lifestyle modification and metformin in obese patients with polycystic ovary syndrome. A randomized, placebo-controlled, double-blind multicentre study. Human Reproduction 2006 21 80-89.

26 Banaszewska B, Duleba AJ, Spaczynski RZ \& Pawelczyk L. Lipids in polycystic ovary syndrome: role of hyperinsulinemia and effects of metformin. American Journal of Obstetrics and Gynaecology 2006 194 1266-1272.

27 Arner P. Free fatty acids - do they play a central role in type 2 diabetes? Diabetes, Obesity and Metabolism 20013 S11-S19.

28 Lebovitz HE \& Banerji MA. Insulin resistance and its treatment by thiazolidinediones. Recent Progress in Hormone Research 200156 265-294.

29 Mori Y, Murakawa Y, Okada K, Horikoshi H, Yokoyama J, Tajima N \& Ikeda Y. Effect of troglitazone on body fat distribution in type 2 diabetic patients. Diabetes Care 199922 908-912.

30 Mitwally MF, Witchel SF \& Casper RF. Troglitazone: a possible modulator of ovarian steroidogenesis. Journal of the Society for Gynecologic Investigations 20029 163-167.

31 Pasquali R. The endocrine of obesity in eumenorrheic women. In Androgen Excess Disorders in Women, pp 507-518. Eds R Azziz, JE Nestler \& D Dewailly, Philadelphia: Lippincott- Raven Publishers, 1997.

32 Dunaif A, Scott D, Finegood D, Quintana B \& Whitcomb R. The insulin-sensitizing agent troglitazone improves metabolic and reproductive abnormalities in the polycystic ovary syndrome. Journal of Clinical Endocrinology and Metabolism $1996 \mathbf{8 1}$ 3299-3306.

33 Azziz R, Ehrmann D, Legro RS, Whitcomb RW, Hanley R, Fereshetian AG, O'Keefe M \& Ghazzi MN. PCOS/Troglitazone Study Group. Troglitazone improves ovulation and hirsutism in the polycystic ovary syndrome: a multicenter, double blind, placebo-controlled trial. Journal of Clinical Endocrinology and Metabolism 200186 1626-1632.

34 Azziz R, Ehrmann DA, Legro RS, Fereshetian AG, O’Keefe M \& Ghazzi MN. PCOS/Troglitazone Study Group. Troglitazone decreases adrenal androgen levels in women with polycystic ovary syndrome. Fertility and Sterility 2003 79 932-937.
35 Cataldo NA, Abbasi F, McLaughlin TL, Lamendola C \& Reaven GM. Improvement in insulin sensitivity followed by ovulation and pregnancy in a woman with polycystic ovary syndrome who was treated with rosiglitazone. Fertility and Sterility $2001 \mathbf{7 6}$ 1057-1059.

36 Ghazeeri G, Kutteh WH, Bryer-Ash M, Haas D \& Ke RW. Effect of rosiglitazone on spontaneous and clomiphene citrate-induced ovulation in women with polycystic ovary syndrome. Fertility and Sterility $2003 \mathbf{7 9} 562-566$.

37 Sepilian V \& Nagamani M. Effects of rosiglitazone in obese women with polycystic ovary syndrome and severe insulin resistance. Journal of Clinical Endocrinology and Metabolism 200590 60-65.

38 Cataldo NA, Abbasi F, McLaughlin TL, Basina M, Fechner PY, Giudice LC \& Reaven GM. Metabolic and ovarian effects of rosiglitazone treatment for 12 weeks in insulin-resistant women with polycystic ovary syndrome. Human Reproduction $2006 \mathbf{2 1}$ 109-120.

39 Rautio K, Tapanainen JS, Ruokonen A \& Morin-Papunen LC. Endocrine and metabolic effects of rosiglitazone in overweight women with PCOS: a randomized placebo-controlled study. Human Reproduction 200621 1400-1407.

40 Majuri A, Santaniemi M, Rautio K, Kunnari A, Vartiainen J, Ruokonen A, Kesaniemi YA, Tapanainenm JS, Ukkola O \& MorinPapunen L. Rosiglitazone treatment increases plasma levels of adiponectin and decreases levels of resistin in overweight women with PCOS: a randomized placebo-controlled study. European Journal of Endocrinology 2007156 263-269.

41 Zawadzki JK \& Dunaif A. Diagnostic criteria for polycystic ovary syndrome. In Polycystic Ovary Syndrome, pp 377-384. Eds A Dunaif, F Haseltine \& GR Merriam, Boston: Blackwell Scientific Publications, 1992.

42 Azziz R. Definition, diagnosis, and epidemiology of the polycystic ovary syndrome. In The Polycystic Ovary Syndrome: Current Concepts on Pathogenesis and Clinical Care, p 5. Ed. R Azziz, New York: Springer Science+Business Media, LCC, 2007.

43 Mavri A, Alessi MC, Bastelica D, Geel-Georgelin O, Fina F, Sentocnik JT, Stegnar M \& Juhan-Vague I. Subcutaneous abdominal, but not femoral fat expression of plasminogen activator inhibitor-1 (PAI-1) is related to plasma PAI-1 levels and insulin resistance and decreases after weight loss. Diabetologia $2001442025-2031$.

44 Matthews DR, Hosker JP, Rudenski AS, Naylor BA, Treacher DF \& Turner RC. Homeostasis model assessment: insulin resistance and beta-cell function from fasting plasma glucose and insulin concentrations in man. Diabetologia $1985 \mathbf{2 8} 412-419$.

45 Hedblad B, Nilsson P, Janzon L \& Berglund G. Relation between insulin resistance and carotid intima-media thickness and stenosis in non-diabetic subjects. Results from a cross-sectional study in Malmo, Sweden. Diabetic Medicine 200017 299-307.

46 Baillargeon JP, Jakubowicz DJ, Iuorno MJ, Jakubowicz S \& Nestler JE. Effects of metformin and rosiglitazone, alone and in combination, in nonobese women with polycystic ovary syndrome and normal indices of insulin sensitivity. Fertility and Sterility 2004 82 893-902.

47 Mitkov M, Pehlivanov B \& Terzieva D. Metformin versus rosiglitazone in the treatment of polycystic ovary syndrome. European Journal of Obstetrics Gynaecology and Reproductive Biology $2006 \mathbf{1 2 6} 93-98$.

48 Legro RS, Zaino RJ, Demers LM, Kunselman AR, Gnatuk CL, Williams NI \& Dodson WC. The effects of metformin and rosiglitazone, alone and in combination, on the ovary and endometrium in polycystic ovary syndrome. American Journal of Obstetrics and Gynaecology 2007196402.

49 Pillai A, Bang H \& Green C. Metformin and glitazones: do they really help PCOS patients? Journal of Family Practice $2007 \mathbf{5 6}$ 444-453.

50 Furnsinn C, Nowotny P, Brunmair B, Gras F, Roden M, Waldhäusl W \& Vierhapper $H$. Thiazolidinediones influence plasma steroids of male obese Zucker rats. Endocrinology 2002 143327. 
51 Vierhapper H, Nowotny P \& Waldhausl W. Reduced production rates of testosterone and dihydrotestosterone in healthy men treated with rosiglitazone. Metabolism 200352 230-232.

52 Gasic S, Nagamani M, Green A \& Urban RJ. Troglitazone is a competitive inhibitor of 3ß-hydroxysteroid dehydrogenase enzyme in the ovary. American Journal of Obstetrics and Gynaecology 2001 184 575-579.

$53 \mathrm{Mu}$ YM, Yanase T, Nishi Y, Waseda N, Oda T, Tanaka A, Takayanagi R \& Nawata H. Insulin sensitizer, troglitazone, directly inhibits aromatase activity in human ovarian granulosa cells. Biochemical and Biophysical Research Communications 2000271 710-713.

54 Arlt W, Auchus RJ \& Miller WL. Thiazolidinediones but not metformin directly inhibit the steroidogenic enzymes P450c17 and 3ß-hydroxysteroid dehydrogenase. Journal of Biological Chemistry 2001276 16767-16771.
55 la Marca A, Egbe TO, Morgante G, Paglia T, Cianci A \& De Leo V. Metformin treatment reduces ovarian cytochrome P-450c17alpha response to human chorionic gonadotrophin in women with insulin resistance-related polycystic ovary syndrome. Human Reproduction 200015 21-23.

56 Balen A. Strategies for ovulation induction in the management of anovulatory polycystic ovary syndrome. In The Polycystic Ovary Syndrome: Current Concepts on Pathogenesis and Clinical Care, $\mathrm{p} 110$. Ed. R Azziz, New York: Springer Science+Business Media, LCC, 2007.

Received 13 February 2008

Accepted 26 February 2008 